tissues. More recently it has been demonstrated that calcitonin, a polypeptide with a molecular weight in the region of 3,000 which lowers the plasma calcium concentration, is also secreted by the thyroid.

At the fifth International Thyroid Conference, which was hold in Rome in 1965, the advances in these and other related subjects which have occurred since the conference held five years previously wore described in a series of papers given by active workers in the field. The book constitutes the proceedings of this conference and the editors are to be warmly congratulated, not only on the enterprise and energy which they have shown in achieving publication so rapidly after the conference, when the work is still fresh, but also in tho coherence which they have achieved in what can be seen to be a somewhat discursive and wide-ranging subject.

There are two excellent reviews, the first, the Dunhill Memorial Lecture, given by S. H. Wollman, entitled "The Heterogeneity of the Thyroid Gland"; and the second, the Van Meter Prize Essay, by J. H. Oppenheimer and G. Bernstein, on "The Metabolism and Physiological Significance of Thyroxine-binding Prealbumin in Man". The papers given on original work are grouped into some twenty-one different sections, and at the end of each the work described is excellently summarized by the closing remarks of the chairman. As at most international conferences, the papers are of variable quality though the general standard is high.

Quite apart from their importance in understanding the activity and disease of the thyroid, these studies are illuminating and creating new approaches in other fields of medicine. For example, a variety of antibodies to a number of antigens within the thyroid have been found not only in Hashimoto's disease but in a number of other much more common thyroid disorders, and theso discoveries have led to the finding of antibodies to other normal body components in pernicious anaemia and in some people with failure of the adrenal cortex; the more-than-chance occurrence of either of these conditions, along with thyroid disease in the same person, had previously been pointed out by clinicians. The finding that Adams and Purves's long-acting thyroid stimulator is a $\gamma_{2}$-globulin, with the properties of an antibody and probably derived from lymphocytes, is a further pointer suggesting the association of a variety of thyroid disorders with some disturbances of immunity. It seems highly likely that the work being carried out in this field will open out the horizons and increase the concepts needed for the general understanding of disease processes.

This well produced book will be invaluable to those who wish to have in one volume a wide survey of all the different aspects of thyroid research which is at present being conducted throughout the world, for there are contributions from twenty-soven different countries. As might be expected, however, anyone who required to study in depth the results and the techniques used in any one of the many facets dealt with would in general need to peruse the original papers in the appropriate sciontific journals.

G. A. Smart

\section{ORGANIC CHEMISTRY OF NITROGEN}

\section{The Chemistry of Open-Chain Organic Nitrogen} Compounds

By Peter A. S. Smith. Vol. 1. Pp. xi+356. \$21.45. Vol. 2: Derivatives of Oxidized Nitrogen: Hydrazines to Nitrates. Pp. xii +531. \$37.50. (New York: W. A. Benjamin, Inc., 1965, 1966.)

Sibgwick's Organic Chemistry of Nitrogen, published in 1910 and revised in 1937, is still a valuable work of reference and a delight to read, but for many years thero has been a clear need for a new comprehensive text sum. marizing in digestible form the chemistry of organic compounds containing nitrogen and providing leading literature references. Prof. Smith has had the courage to undertake this task, and in these two volumes he has succeeded admirably.

The fifteen chapters are concerned successively with a survey of inorganic nitrogen compounds; aliphatic amines; aromatic amines; amides; nitriles and isocyanides; nitrogen derivatives of carbonic acid; ammonia derivatives of the carbonyl group; hydroxylamine derivatives; hydrazine derivatives; azides and diazo com. pounds; diazonium, azo, and azoxy compounds; compounds with chains of three or more nitrogens; C-nitroso compounds; C-nitro compounds; esters and amides of nitrogen oxy-acids. Some chapters inevitably are sub. divided, hydrazine derivatives, for instance, being dis. cussed under alkyl and aryl hydrazines, hydrazones, and hydrazides.

Following an account of structure and physical properties, the main body of each chapter or sub-section is devoted to reactions, classified and discussed with emphasis on mechanism. The leisurely style adopted by Sidgwick and others is, unfortunately, no longer possible, but the mechanism and limitations of selected reactions of special practical importance, for example, the deamination of aliphatic amines, the Hofmann and Cope eliminations, and the Beckmann, benzidine and Curtius rearrangements, are discussed in greater detail. Finally, preparative methods are summarized and sources of typical experimental procedures are indicated. There are approximately four thousand references designed to cover key papers, review articles and especially the recent literature up to 1964 , with the mention of some more recent publications. The author is wise in omitting heterocyclic compounds and alkaloids, since both subjects have received exhaustive and continuous attention in books and reviews. Openchain derivatives, on the other hand, have been neglected previously; the less well known and often obscurely named groups, for example amidrazones, nitramines, hydroxamic acids, urethans and sulphonamides, are difficult to trace in the literature, and the systematic accounts of these compounds should prove to be of particular value.

It is a rare pleasure to read books of this type written by a single author. In this way the faults of the multiauthor compendium are avoided, particularly the lack of a unified approach, and tho hold-up of certain chapters resulting sometimes in the remainder becoming out of date even before publication. These volumes should be purchased by every chemical library; it is regrettable that the high price will prevent many individuals interested in the organic chemistry of nitrogen from acquiring personal copies.

M. F. GRUNDON

\section{NON-EQUILIBRIUM BIOLOGY}

\section{Nonequilibrium Thermodynamics in Biophysics}

By A. Katchalsky and Peter F. Curran. (Harvard Books in Biophysios, No. 1.) Pp. $x+848$. (Cambridge, Mass.: Harvard University Press; London: Oxford University Press, 1965.) 68s. net.

"Livrng systems are non-equilibrium, open systems in which irreversible processes are occurring...". This statement in the authors' introduction provides the fundamental justification for the writing of this book. Indeed, the realm of biological systems is the field in which the relatively new science of non-equilibrium thermodynamics (NET) finds the most beautiful applications. It is disappointing, however, to see that relatively very few biological problems have been attacked by using the methods of this discipline, and the number of open problems is still enormous. This abnormal situation is mainly due to the fact that many biologists just ignore the possibilities (or even the existence!) of NET. For this reason the 